\title{
Restoration of chaperone-mediated autophagy in aging liver improves cellular maintenance and hepatic function
}

\author{
Cong Zhang and Ana Maria Cuervo \\ Department of Developmental and Molecular Biology and Department of Anatomy and Structural \\ Biology, Marion Bessin Liver Research Center and Institute for Aging Research, 1300 Morris Park \\ Avenue, Albert Einstein College of Medicine, Bronx, New York 10461, USA.
}

\begin{abstract}
Chaperone-mediated autophagy (CMA), a selective mechanism for degradation of cytosolic proteins in lysosomes, contributes to the removal of altered proteins as part of the cellular quality-control systems ${ }^{1,2}$. We have previously found that CMA activity declines in aged organisms and have proposed that this failure in cellular clearance could contribute to the accumulation of altered proteins, the abnormal cellular homeostasis and, eventually, the functional loss characteristic of aged organisms. To determine whether these negative features of aging can be prevented by maintaining efficient autophagic activity until late in life, in this work we have corrected the CMA defect in aged rodents. We have generated a double transgenic mouse model in which the amount of the lysosomal receptor for CMA, previously shown to decrease in abundance with age ${ }^{3}$, can be modulated. We have analyzed in this model the consequences of preventing the age-dependent decrease in receptor abundance in aged rodents at the cellular and organ levels. We show here that CMA activity is maintained until advanced ages if the decrease in the receptor abundance is prevented and that preservation of autophagic activity is associated with lower intracellular accumulation of damaged proteins, better ability to handle protein damage and improved organ function.
\end{abstract}

\begin{abstract}
Autophagy is a cellular process that mediates the degradation of intracellular components in lysosomes, thus contributing to maintenance of cellular homeostasis, intracellular clearance of damaged structures and adaptation to environmental challenges ${ }^{4}$. Defective autophagy has been linked to common human diseases ${ }^{4}$. A decrease in autophagic activity with age, described in almost all model organisms analyzed, has been proposed to contribute to age-dependent accumulation of damaged intracellular components that lead to altered cellular homeostasis and loss of function in aging 5 .

Three different autophagic pathways - macroautophagy, microautophagy and CMA—have been described in mammalian cells on the basis of their mechanisms for delivery of cargo to lysosomes ${ }^{4,6}$. Whereas in macro- and microautophagy complete regions of the cytosol are sequestered and delivered to lysosomes all at once, in CMA individual proteins cross the lysosomal membrane one by one for their degradation ${ }^{1,2}$. The substrates of CMA are a subset of cytosolic proteins with a motif recognized by the hsc 70 chaperone ${ }^{7}$. The chaperone-substrate complex binds to the CMA receptor, the lysosomal-associated membrane protein-2A
\end{abstract}

(C) 2008 Nature Publishing Group

Correspondence should be addressed to A.M.C. (amcuervo@aecom.yu.edu). AUTHOR CONTRIBUTIONS

C.Z. performed all the experiments in this study. A.M.C. conceived, designed and directed the study.

Published online at http://www.nature.com/naturemedicine/

Reprints and permissions information is available online at http://npg.nature.com/reprintsandpermissions/

Note: Supplementary information is available on the Nature Medicine website. 
(LAMP-2A $)^{8}$. After unfolding ${ }^{9}$, the substrate crosses the lysosomal membrane assisted by a lumenal chaperone (lys-hsc70) ${ }^{10}$ and is rapidly degraded. CMA is maximally activated during stresses such as prolonged starvation, mild oxidation and other conditions resulting in protein damage $^{1,2}$. CMA activity decreases during aging ${ }^{3}$ and in some age-related disorders such as familial forms of Parkinson's disease ${ }^{11}$.

We have proposed that reduced lysosomal abundance of LAMP-2A is responsible for the decline in CMA activity during aging ${ }^{3}$. To determine whether maintaining LAMP-2A abundance constant throughout the mouse life span prevents autophagic decline and delays aging features associated with poor handling of cellular damage, we generated a double transgenic mouse carrying a transgene encoding a Tet regulator (which is bound by tetracycline or a related antibiotic, doxycycline) under the control of the albumin promoter (Alb-Tet-offL2A). In this mouse, expression of an exogenous copy of the gene encoding LAMP-2A can be regulated in liver-where the age-related CMA defect has been well characterized ${ }^{3,12}$-by addition of doxycycline to the diet (doxycycline diet; Fig. 1a). In young Alb-Tet-off-L2A mice, we verified that removal of the doxycycline diet increased LAMP-2A abundance two- to fourfold only in liver, that LAMP-2A was properly targeted to lysosomes and did not alter the levels of other LAMPs, and that the additional LAMP-2A was functional in CMA, as lysosomal-enriched fractions isolated from young transgenic mice exposed to mild oxidative stress (to maximally activate CMA) showed higher rates of CMA than those from wild-type littermates (Fig. 1b-d and Supplementary Fig. 1 online). Expression of the Tet regulator in liver was mainly restricted to hepatocytes (Supplementary Fig. 2 online).

A decrease in LAMP-2A abundance becomes evident in mouse liver at 9-12 months of age as a result of the increased instability of this protein at the lysosomal membrane ${ }^{3,12}$. Given that our intention was to maintain LAMP-2A throughout the mouse life span at levels found in young adults, rather than to upregulate CMA from early on in life, we repressed the expression of the transgene until 6 months of age, when we switched the mice to a doxycycline-free diet until they were 22-26 months old (early activation; Fig. 1e). At that age, the amount of LAMP-2A in liver lysosomes from the Alb-Tet-off-L2A mice was comparable to that detected in 6-month-old wild-type mice and was significantly $(P<0.001)$ higher than in wild-type or transgenic littermate mice maintained with a doxycycline diet throughout their life span (Fig. 1f). Accordingly, lysosomes from 22-month-old transgenic mice showed rates of uptake of a well characterized CMA substrate similar to those in lysosomes from 6-month-old wild-type mice (Fig. 1g), thus supporting preserved CMA activity in these mice. It is noteworthy that although the age-dependent decline in LAMP-2A abundance does not result from downregulation of expression of the transgene ${ }^{12}$, the amount of LAMP-2A in the aged mice was similar to but not higher than that in the young wild-type mice (Fig. 1g). This disparity between LAMP-2 mRNA and protein levels is a consequence of the previously described instability of the protein in the membrane of aged liver lysosomes ${ }^{12}$. However, the disparity worked in our favor in this model, because our intention was not to upregulate CMA in aged mice but rather to maintain the activity of the pathway at the levels observed in the young mice.

To determine whether CMA activity could be restored once the age-dependent decline had already occurred, in a second group of transgenic mice we activated the expression of the extra copy of LAMP-2A only 3 months before we killed them (late activation; Fig. 1e). LAMP-2A abundance in these mice was similar to that observed in the early activated group and was markedly higher than in wild-type littermates (Fig. 1h). Incubation of intact isolated lysosomes with a radiolabeled CMA substrate in vitro (to recapitulate binding, uptake and degradation of the substrates via CMA) revealed increased CMA activity ( $24 \%$ increase), but to a lower extent than in the early activated group ( $46 \%$ increase; Fig. 1i). Higher rates of substrate proteolysis in the transgenic mice were mostly due to increased binding and uptake by CMA, as this increase was no longer evident when the lysosomal membrane was disrupted to grant access 
of the lysosomal enzymes to the substrate (Fig. 1i). In fact, most of the previously described changes with age in abundance and activity of lysosomal proteases in liver do not seem to affect the subpopulation of lysosomes responsible for $\mathrm{CMA}^{3}$. Thus, we did not find substantial differences in the levels and activities of two major lysosomal proteases, cathepsin B and cathepsin D, in these lysosomes in any of the groups of mice analyzed (Supplementary Fig. 3 online). These results confirm that reduced amounts of LAMP-2A with age are a primary cause for CMA decline and that restoration of normal amounts of LAMP-2A, even for a short period, results in improved CMA activity.

Analysis of lysosomal chaperone abundance revealed that the compensatory increase in hsc70 previously described in lysosomes from aged rodents ${ }^{3}$ was still evident in the lysosomes isolated from aged transgenic mice (Supplementary Fig. 4 online). Notably, upregulation was observed only for the lumenal form of hsc70, which could contribute to the enhanced CMA activity, whereas the amount of membrane-associated hsc70 in the aged transgenic mice was indistinguishable from that found in young wild-type mice (Supplementary Fig. 4). The agerelated changes in hsp90 chaperone abundance at the lysosomal membrane and lumen and of hsp40 at the membrane were not corrected in the aged transgenic mice (Supplementary Discussion online).

We next examined the cellular consequences of preserving normal CMA activity until advanced age in the livers of the Alb-Tet-off-L2A transgenic mice. In light of the proposed role for CMA in the removal of altered proteins ${ }^{13,14}$, we first compared levels of oxidized proteins in cytosolic liver fractions from the different mouse groups. As previously described $^{3}$, levels of oxidized proteins (detected with an antibody to carbonyl groups after twodimensional electrophoresis) were markedly increased in liver cytosol from 22-month-old wild-type mice when compared with 6-month-old mice (Fig. 2a). Of note, the amount of oxidized proteins was drastically reduced in liver cytosol from both groups of transgenic mice (Fig. 2a). The reduced content of oxidized proteins was restricted to the liver, and it was not observed if the transgene was repressed throughout the life span (Fig. 2a and Supplementary Fig. 5 online). Similar results were observed for proteins modified by 4-hydroxynonenal, a toxic product of lipid peroxidation and a sensitive marker of oxidative damage (Fig. 2b). In support of our hypothesis that the lower levels of oxidized proteins in the cytosol of the aged transgenic mice are, at least in part, a consequence of their improved removal by CMA, we found a higher content of oxidized substrates in the lumen of CMA-active lysosomes from the aged transgenic mice when compared with wild-type littermates (Supplementary Fig. 6 online). We then compared the aggregate protein content in livers of the different groups of mice with an antibody to ubiquitin, as protein aggregates are enriched in polyubiquitinated chains. The amount of aggregates detected as polyubiquitinated proteins retained in the stacking of the gel after electrophoresis (data not shown) or directly after immunostaining of liver sections was significantly $(P<0.05)$ lower in the livers of the aged transgenic mice when compared to wildtype littermates (Fig. 2c).

Comparative morphological analysis revealed higher ultrastructural similarity between the livers of aged transgenic mice and those of young wild-type mice than with those of aged wildtype mice (Fig. 3a). In agreement with previous reports in other strains ${ }^{15}$, hepatocytes from our aged wild-type mice presented a higher content (in number and size) of lipid droplets, increased vesiculation and marked changes in organelle morphology (endoplasmic reticulum often accumulated as cisterna stacks, and mitochondria had reduced density, were swollen and had deformed cristae; Fig. 3a). These changes were no longer detectable in the early activated transgenic mice and were considerably attenuated in the late activated group (Fig. 3a).

Preserved mitochondrial structure is associated with functional improvement. Thus, the intracellular ATP content, the activity of mitochondrial succinic dehydrogenase (reported to decrease with age) and the permeability of the mitochondrial membrane were all closer between 
young wild-type mice and the two groups of older transgenic mice than they were with the older wild-type littermates (Supplementary Fig. 7 online). Both the lower content of damaging cytosolic products (that is, oxidized and aggregate proteins) in the Alb-Tet-off-L2A mouse livers as well as improved organelle turnover could help to preserve functional mitochondria in these mice. In fact, analysis of several different indirect markers suggests that these mice also showed improved macroautophagic activity. Reduced autophagy of mitochondria is in part responsible for mitochondrial dysfunction in aged organisms ${ }^{16}$. In agreement with previous reports, we observed extensive accumulation of double-membrane vesicles with recognizable cargo (autophagosomes) in livers of aged wild-type mice (Fig. 3b), which has been attributed to problems in autophagosome clearance by lysosomes that increase with age ${ }^{17}$. In contrast, the ratio of autophagosomes (before lysosomal fusion) to autophagolysosomes (after lysosomal fusion) in the early activated aged transgenic mice was closer to that detected in young mice (Fig. 3b). Although to a lesser degree, late activation of the transgene also improved macroautophagic flow. Higher amounts of the macroautophagy effector Atg5-Atg12 and lower amounts of LC3-II, an autophagosome marker, in the aged transgenic mice livers were also indirect evidence of more efficient macroautophagy activation and autophagosome clearance, respectively, in these mice (Supplementary Fig. 8 online). Furthermore, the characteristic decrease in the amount of total liver proteins after $6 \mathrm{~h}$ of starvation, which is mostly due to the bulk degradation of proteins and organelles by macroautophagy, was markedly reduced in aged wild-type mice but not in transgenic littermates (Supplementary Fig. 8d). These results indirectly support preservation of appropriate starvation-induced macroautophagy in the livers of the aged transgenic mice. Lastly, livers of aged Alb-Tet-off-L2A mice showed lower levels of lipofuscin, the autofluorescent aging pigment originating from undegraded damaged products (mostly mitochondria) inside various lysosomal compartments ${ }^{18}$ (Fig. 3c and Supplementary Discussion). In support of a generalized beneficial effect of preserving functional CMA in intracellular clearance, livers of aged Alb-Tet-off-L2A mice also showed a delay in the agedependent changes in the ubiquitin-proteasome system. The decrease in the three catalytic activities of the $20 \mathrm{~S}$ proteasome, the changes in the electrophoretic properties of the $\beta$-subunits of this protease and the increased amounts of cytosolic polyubiquitinated proteins observed in aged wild-type mouse livers were no longer evident in the aged transgenic mice (Supplementary Fig. 9 online). The improved functioning of the different proteolytic systems supports the existence of cross-talk among them that could be used to preserve their function until advanced age (Supplementary Discussion).

We next examined the consequences of improved cellular homeostasis at the organ level in the aged transgenic mice. Overall, signs of cellular damage were reduced in livers of the Alb-Tetoff-L2A mice until advanced ages. Both serum abundance of alanine aminotransferase (Fig. 4a), a well established marker of liver injury ${ }^{19}$, and the number of apoptotic cells (detected by TUNEL assay or by caspase- 3 activation ${ }^{20}$ (Fig. 4b,c and Supplementary Fig. 10 online) were markedly lower in aged transgenic mice than in their wild-type littermates. The lower serum alanine aminotransferase abundance did not reflect changes in the liver inflammatory state, as the number and size of infiltrates were comparable in aged wild-type and transgenic mice (Supplementary Fig. 11 online). These results support our hypothesis that the efficient handling of altered proteins in the transgenic mice has a positive impact on hepatocyte viability.

Lastly, we analyzed whether or not the preserved autophagic activity in the liver of aged transgenic mice had any effect on liver function. Using the zoxazolamine-induced paralysis time assay ${ }^{21}$, a common test for liver function, we found significant $(P<0.001)$ shortening in the time required for the aged transgenic mice to metabolize the injected muscle relaxant, reaching recovery times indistinguishable from those in young wild-type mice (Fig. 4d). Although this marked functional improvement surpassed our initial predictions, we do not think that a single protein, LAMP-2A, is responsible for the decline in liver function with age. 
Instead, we argue that our findings support the idea that restoration of one of the cellular quality control mechanisms - in this case, CMA — improves the intracellular milieu (by preventing accumulation of damaged proteins), and this slows down the deterioration of the other quality control mechanisms (Supplementary Discussion). This improved functioning of all the clearance systems has an amplifying beneficial effect that manifests as improvement in cellular homeostasis, organelle status and ability to buffer cellular damage in the Alb-Tet-off-L2A mice, which ultimately results in better organ functioning.

In conclusion, to our knowledge, this work shows for the first time in vivo that maintenance of proper autophagic activity throughout life span prevents or slows down the functional failure associated with cellular proteotoxicity and accumulation of intracellular damage in aging.

\section{METHODS}

\section{Chemicals and antibodies}

We acquired reagents and antibodies from sources described previously $3,8,13,22$. Antibody to the $\beta$ subunits of the $20 \mathrm{~S}$ proteasome was from Biomol, antibody to ubiquitin was from Invitrogen, antibodies to cathepsin B and cathepsin D were from Santa Cruz, antibody to the Tet repressor was from Genlantis, antibody to cleaved caspase-3 was from Cell Signaling Technology, antibody to CD31 (PECAM-1) was from BD Pharmingen, antibody to hydroxynonenal was from Abcam and antibodies to LC3 and Atg5-Atg12 were from Novus Biologicals. We detected carbonyl groups in oxidized proteins with the OxyBlot Protein Oxidation Detection Kit from Chemicon International.

\section{Generation of a liver-specific Tet-off-LAMP-2A double transgenic mouse}

We generated the transgenic TRE-LAMP-2A mouse in an FVB background by microinjection of embryonic stem cells transfected with the mouse Lamp2a cDNA downstream from the tetracycline response element (TRE, a tetracycline-controlled transactivator (tTA)-dependent promoter) in the pUHD10-3 plasmid. We generated the liver-specific Tet-off-LAMP-2A double transgenic mouse line by crossing homozygous TRE-LAMP-2A mice with mice carrying tTA under the control of the albumin promoter (Alb-tTA mice; Fig. 1a). We confirmed the presence of TRE-LAMP-2A and Alb-tTA in the double transgenic mice by both Southern blot and PCR analyses (Supplementary Fig. 1). We repressed expression of the transgene during gestation and before weaning by maintaining the mothers on a doxycyclinesupplemented diet ( $200 \mathrm{mg} \mathrm{kg}^{-1}$ doxycycline; Bioserve), as doxycycline can cross the placenta and also gets secreted with the milk. After weaning, we maintained all of the mice on the doxycycline diet until we wanted to activate the transgene. All results presented in this study are the mean values of data obtained with mice from two founder lines with similar expression (to discard insertion-related effects) and are normalized for the amount of LAMP-2A expression in each individual mouse. We used male mice for all studies, except for the zoxazolamine-induced paralysis test in which we separately analyzed males and females and ultimately pooled the obtained values as there were no significant differences between genders. We switched wild-type and transgenic mice to a doxycycline-free diet at 6 months of age (early activation) or 3 months before use (late activation). We initially kept results with wild-type and transgenic mice in the 6-month-old group separate, but because we did not find any difference in LAMP-2A abundance or in any of the other parameters analyzed between both groups, for simplification, we pooled results obtained from these two groups into a single wildtype 6-month-old group. We used the same approach for the group of 22-month-old wild-type mice and the group of transgenic mice maintained on the doxycycline food throughout their lives, which we pooled together as a single wild-type 22-month-old group. We did not find differences in the aging phenotype that could be attributed to the long-term feeding of doxycycline food in the wild-type mice. Thus, standard biochemical tests for the analysis of 
cellular homeostasis (alanine aminotransferase and ATP abundance, protein aggregation, lipofuscin content and mitochondria function) and routine analysis of phenotypic changes of aging (global health status, spontaneous tumor incidence, weight, degree of kyphosis, skin color and hair loss) did not reveal significant differences between wild-type mice maintained on regular food and those on doxycycline food for 22 months. Because male FVB mice in our facilities have a maximum life span of 30 months but have a median life span of 19 months, we selected the age range of 22-26 months as the end point in these studies to guarantee that the mice were not close to terminal conditions, at which point unrelated factors could make the interpretation of results difficult. All animal studies were approved by the Animal Care Committee at the Albert Einstein College of Medicine.

\section{Isolation of lysosomal fractions}

We isolated mouse liver lysosomes from a light mitochondrial-lysosomal fraction in a discontinuous metrizamide density gradient ${ }^{23}$. The purity of the lysosomal fractions was equivalent in the four groups of mice (6- and 22-month-old wild-type mice and 22-month-old early-and late-activated Alb-Tet-off-L2A mice), as determined by the recovery of common lysosomal enzymes in the fractions (6-10\% of total $\beta$-hexosaminidase activity was recovered in isolated lysosomes with an enrichment of 20-35-fold). In all of the experiments, we verified the lysosomal integrity after isolation by measuring the activity of $\beta$-hexosaminidase, a lysosomal enzyme, in the incubation medium ${ }^{24}$. We discarded preparations containing more than $10 \%$ broken lysosomes immediately after isolation. We prepared the cytosolic fraction by centrifugation of the supernatant of the light mitochondrial-lysosomal fraction at $100,000 \mathrm{~g}$ for $1 \mathrm{~h}$ at $4{ }^{\circ} \mathrm{C}$.

\section{Uptake of substrate proteins by isolated lysosomes}

We analyzed the transport of purified proteins into isolated lysosomes using a previously described in vitro system ${ }^{3,8,13,22}$. Briefly, we incubated glyceraldehyde 3-phosphate dehydrogenase (GAPDH) or RNase A with freshly isolated mouse liver lysosomes in MOPS buffer (10 mM 3-( $N$-morpholino) propanesulfonic acid pH 7.3, $0.3 \mathrm{M}$ sucrose) for $20 \mathrm{~min}$ at $37^{\circ} \mathrm{C}$. Where indicated, we preincubated lysosomes with a cocktail of protease inhibitors (10 $\mathrm{mM}$ leupeptine, $10 \mathrm{mM}$ 4-(2-aminoethyl) benzenesulfonyl fluoride hydrochloride, $1 \mathrm{mM}$ pepstatin, $100 \mathrm{mM}$ EDTA) for $10 \mathrm{~min}$ at $0{ }^{\circ} \mathrm{C}$. At the end of the incubation, we collected the lysosomes by centrifugation, washed them with MOPS buffer and subjected them to SDSPAGE and immunoblotting with antibodies specific for the assayed protein. We calculated transport by subtracting from the amount of protein associated with lysosomes treated with protease inhibitors (protein bound to the lysosomal membrane and taken up by lysosomes) the amount of protein associated with untreated lysosomes (protein bound to the lysosomal membrane), as previously described ${ }^{9,13}$. In the absence of protease inhibitors, the substrate that reaches the lysosomal lumen is rapidly degraded and the only substrate remaining is that bound to the lysosomal membrane. If lysosomal proteases are blocked, both the membranebound protein and that translocated into the lumen can be detected. Uptake is calculated as the difference between the amount of substrate associated with protease inhibitor-treated lysosomes and the amount bound to the lysosomal membrane without protease inhibitors.

\section{Degradation of proteins by isolated lysosomes}

We assayed lysosomal degradation of exogenously added proteins as described before ${ }^{13,25}$ by incubation of intact lysosomes with GAPDH or RNase A radiolabeled by reductive methylation ${ }^{26}$ in MOPS buffer supplemented with $1 \mathrm{mM}$ DTT and 5.6 $\mu \mathrm{M}$ cysteine. We stopped reactions with a final concentration of $10 \%$ trichloroacetic acid, and, after filtration in the Millipore Multiscreen Assay System (Millipore) with a $0.22-\mu \mathrm{m}$ pore membrane, we measured radioactivity in the flow-through in a WinSpectral 1414 liquid scintillation counter (Perkin 
Elmer). We expressed proteolysis as the percentage of the initial acid-precipitable radioactivity (protein) transformed into acid-soluble radioactivity (amino acids and small peptides) during the incubation time. We assayed degradation of $\left[{ }^{14} \mathrm{C}\right]$ labeled substrates by disrupted lysosomes using a similar procedure but carried out the incubation in water in order to leave the lumenal lysosomal $\mathrm{pH}$ undisturbed.

\section{Transmission electron microscopy}

We fixed liver blocks $\left(1 \mathrm{~mm}^{3}\right.$ in size) with $2 \%$ paraformaldehyde and $2.5 \%$ gluteraldehyde in $0.1 \mathrm{M}$ sodium cacodylate buffer, postfixed them with $1 \%$ osmium tetroxide followed by $1 \%$ uranyl acetate, dehydrated them through a graded series of ethanol washes and embedded them in LX112 resin (LADD Research Industries). We cut ultrathin (80-nm) sections on a Reichert Ultracut UCT, stained the sections with uranyl acetate followed by lead citrate and viewed them on a JEOL 1200EX transmission electron microscope at $80 \mathrm{kV}$.

\section{Histological procedures}

For histological staining, we fixed the liver specimens in $10 \%$ neutral formalin and stained 6$\mu \mathrm{m}$ sections with H\&E, periodic acid-Schiff stain or Masson's trichrome stain. A pathologist examined the tissue sections in a blinded fashion and scored them for degree of liver inflammation. For immunohistochemistry, we took deparaffinized and unstained liver sections following standard procedures for epitope retrieval, quenched them and blocked them before incubation with the desired primary antibodies. We then washed the slides extensively and, upon incubation with fluorescence-conjugated secondary antibodies, mounted them in SlowFade Gold antifade reagent with DAPI (Invitrogen). We then examined them on an Axiovert 200 fluorescence microscope (Zeiss). For visualization of lipofuscin deposits, we observed unstained liver sections directly under the fluorescence microscope through the Texas Red light channel. We used Image $\mathbf{J}$ software (US National Institutes of Health) to quantify the number of particles, size and cellular area occupied in 15-20 different randomly taken micrographs for each condition after thresholding. For TUNEL, we stained the free 3 '-OH termini of double- or single-stranded, low molecular weight DNA fragments, resulting from cleavage of genomic DNA during apoptosis, in deparaffinized and rehydrated liver sections using a commercial kit (Roche Applied Science) according to the manufacturer's instructions. We visualized stained sections under the fluorescence microscope.

\section{Zoxazolamine induced paralysis test}

We analyzed the clearance time of zoxazolamine, a muscle relaxant, as an index of liver function ${ }^{21}$. We gave mice a single intraperitoneal injection of zoxazolamine $\left(150 \mathrm{mg} \mathrm{kg}^{-1}\right.$, in olive oil), placed them on their backs and recorded the time required to regain righting reflex after the paralysis induced by this compound.

\section{General methods}

We determined the protein concentration by the Lowry method using BSA as a standard ${ }^{27}$. We used the CellTiter-Glo Assay Kit (Promega) to quantify the amount of ATP in liver homogenates (we detected luminescent signal under a luminometer with an integration time of $1 \mathrm{~s}$ per well). We measured the alanine aminotransferase activity in serum samples by a colorimetric assay using a commercial kit (TECO Diagnostics). We determined cathepsin B activity by measuring the fluorescent signal of 7-amido-4-methylcoumarin (7-AMC) resulting from the hydrolysis of the fluorogenic substrate Arg-Arg-7-AMC ${ }^{28}$. We used cleavage of hemoglobin, monitored as the absorbance soluble in acid at $280 \mathrm{~nm}$, to determine cathepsin D activity in the liver samples ${ }^{28}$. We determined the trypsin-like, chymotrypsin-like and peptidylglutamyl-peptide hydrolase activities of the $20 \mathrm{~S}$ proteasome by measuring the fluorescence of 7-AMC in different C-terminal 7-AMC-containing peptides (Sigma-Aldrich). 
We followed mitochondrial swelling in response to a $50 \mu \mathrm{M}$ calcium challenge by measuring changes in the light absorbance at $540 \mathrm{~nm}$ of mitochondrial suspensions for $10 \mathrm{~min}$ at $25^{\circ} \mathrm{C}$. We performed isoelectric focusing (IEF) with the Protean IEF Cell (Bio-Rad) at $20^{\circ} \mathrm{C}$ with rapid ramping to a voltage of $10,000 \mathrm{~V}$ at a current limit of $50 \mu \mathrm{A}$ using ReadyStrip IPG Strips with a nonlinear 3-10 $\mathrm{pH}$ range (Bio-Rad). We used SYPRO Ruby Protein Stain (Bio-Rad) to highlight total proteins in one-and two-dimensional gels. After SDS-PAGE and immunoblotting, we visualized the proteins recognized by the specific antibodies by standard chemiluminescence methods (Renaissance, NEN-Life Science Products). We exposed membranes to BioMax Light Kodak films (Kodak) for increasing periods of time ranging from $5 \mathrm{~s}$ to $10 \mathrm{~min}$. We performed densitometric quantification of the immunoblotted membranes with an Image Analyzer System (Inotech S-100). We quantified the intensity of the bands with the square 'spot denso' routine of the Image Analyzer System.

\section{Statistical analysis}

Results are shown as the means \pm s.e.m. We first analyzed the differences among the groups by analysis of variance followed by post hoc Bonferroni analysis to detect significant differences between individual groups. We considered a value of $P<0.05$ statistically significant. We performed statistical analysis with GraphPad InStat software (GraphPad).

\section{Supplementary Material}

Refer to Web version on PubMed Central for supplementary material.

\section{ACKNOWLEDGMENTS}

We are grateful to S. Kaushik and A.C. Massey for assistance in the preparation of this manuscript. The pUHD10-3 plasmid and the Alb-tTA mice were generous gifts from H. Bujard (University of Heidelberg) and the antibody to the asialoglycoprotein receptor was kindly supplied by A. Wolkoff (Albert Einstein College of Medicine). This work was supported in part by US National Institutes of Health National Institute on Aging grants AG021904 and AG19834, National Institute of Diabetes and Digestive and Kidney Diseases DK041918, an Ellison Medical Foundation Award and a Glenn Foundation Award.

\section{References}

1. Dice JF. Chaperone-mediated autophagy. Autophagy 2007;3:295-299. [PubMed: 17404494]

2. Massey AC, Zhang C, Cuervo AM. Chaperone-mediated autophagy in aging and disease. Curr. Top. Dev. Biol 2006;73:205-235. [PubMed: 16782460]

3. Cuervo AM, Dice JF. Age-related decline in chaperone-mediated autophagy. J. Biol. Chem 2000;275:31505-31513. [PubMed: 10806201]

4. Mizushima N, Levine B, Cuervo A, Klionsky D. Autophagy fights disease through cellular selfdigestion. Nature 2008;451:1069-1075. [PubMed: 18305538]

5. Cuervo AM, et al. Autophagy and aging: the importance of maintaining 'clean' cells. Autophagy 2005;1:131-140. [PubMed: 16874025]

6. Cuervo AM. Autophagy: in sickness and in health. Trends Cell Biol 2004;14:70-77. [PubMed: 15102438]

7. Chiang HL, Terlecky SR, Plant CP, Dice JF. A role for a $70 \mathrm{kDa}$ heat shock protein in lysosomal degradation of intracellular protein. Science 1989;246:382-385. [PubMed: 2799391]

8. Cuervo AM, Dice JF. A receptor for the selective uptake and degradation of proteins by lysosomes. Science 1996;273:501-503. [PubMed: 8662539]

9. Salvador N, Aguado C, Horst M, Knecht E. Import of a cytosolic protein into lysosomes by chaperonemediated autophagy depends on its folding state. J. Biol. Chem 2000;275:27447-27456. [PubMed: 10862611]

10. Agarraberes FA, Terlecky SR, Dice JF. An intralysosomal hsp70 is required for a selective pathway of lysosomal protein degradation. J. Cell Biol 1997;137:825-834. [PubMed: 9151685] 
11. Cuervo AM, Stefanis L, Fredenburg R, Lansbury PTJ, Sulzer D. Impaired degradation of mutant $\alpha$ synuclein by chaperone-mediated autophagy. Science 2004;305:1292-1295. [PubMed: 15333840]

12. Kiffin R, et al. Altered dynamics of the lysosomal receptor for chaperone-mediated autophagy with age. J. Cell Sci 2007;120:782-791. [PubMed: 17284523]

13. Kiffin R, Bandyopadhyay U, Cuervo AM. Oxidative stress and autophagy. Antioxid. Redox Signal 2006;8:152-162. [PubMed: 16487049]

14. Finn PF, Dice JF. Ketone bodies stimulate chaperone-mediated autophagy. J. Biol. Chem 2005;280:25864-25870. [PubMed: 15883160]

15. Schmucker DL. Age-related changes in liver structure and function: Implications for disease? Exp. Gerontol 2005;40:650-659. [PubMed: 16102930]

16. Cavallini G, Donati A, Taddei M, Bergamini E. Evidence for selective mitochondrial autophagy and failure in aging. Autophagy 2007;3:26-27. [PubMed: 16963838]

17. Terman A. The effect of age on formation an elimination of autophagic vacuoles in mouse hepatocyte. Gerontology 1995;41:319-325. [PubMed: 8821342]

18. Brunk UT, Terman A. Lipofuscin: mechanisms of age-related accumulation and influence on cell function. Free Radic. Biol. Med 2002;33:611-619. [PubMed: 12208347]

19. Tanaka K, Nanbara S, Tanaka T, Koide H, Hayashi T. Aminotransferase activity in the liver of diabetic mice. Diabetes Res. Clin. Pract 1988;5:71-75. [PubMed: 3402335]

20. Higami Y, et al. Aging accelerates but life-long dietary restriction suppresses apoptosis-related Fas expression on hepatocytes. Am. J. Pathol 1997;151:659-663. [PubMed: 9284813]

21. Pappas P, Stephanou P, Vasiliou V, Marselos M. Zoxazolamine-induced paralysis in two rat substrains: differences in hepatic drug metabolism. Eur. J. Drug Metab. Pharmacokinet 1998;23:461467. [PubMed: 10323328]

22. Massey AC, Kaushik S, Sovak G, Kiffin R, Cuervo AM. Consequences of the selective blockage of chaperone-mediated autophagy. Proc. Natl. Acad. Sci. USA 2006;103:5805-5810. [PubMed: $16585521]$

23. Wattiaux R, Wattiaux-De Coninck S, Ronveaux-Dupal M, Dubois F. Isolation of rat liver lysosomes by isopycnic centrifugation in a metrizamide gradient. J. Cell Biol 1978;78:349-368. [PubMed: 211139]

24. Storrie B, Madden EA. Isolation of subcellular organelles. Methods Enzymol 1990;182:203-225. [PubMed: 2156127]

25. Agarraberes FA, Terlecky SR, Dice JF. An intralysosomal hsp70 is required for a selective pathway of lysosomal protein degradation. J. Cell Biol 1997;137:825-834. [PubMed: 9151685]

26. Jentoft N, Dearborn D. Protein labeling by reductive alkylation. Methods Enzymol 1983;91:570-579. [PubMed: 6855602]

27. Lowry OH, Rosebrough NJ, Farr AL, Randall RJ. Protein measurement with the Folin phenol reagent. J. Biol. Chem 1951;193:265-275. [PubMed: 14907713]

28. Barret, A. Handbook of Proteolytic Enzymes. Barrett, AJ.; Rawlings, ND.; Woessner, JF., editors. San Diego: Academic Press; 1998. p. 850-862. 

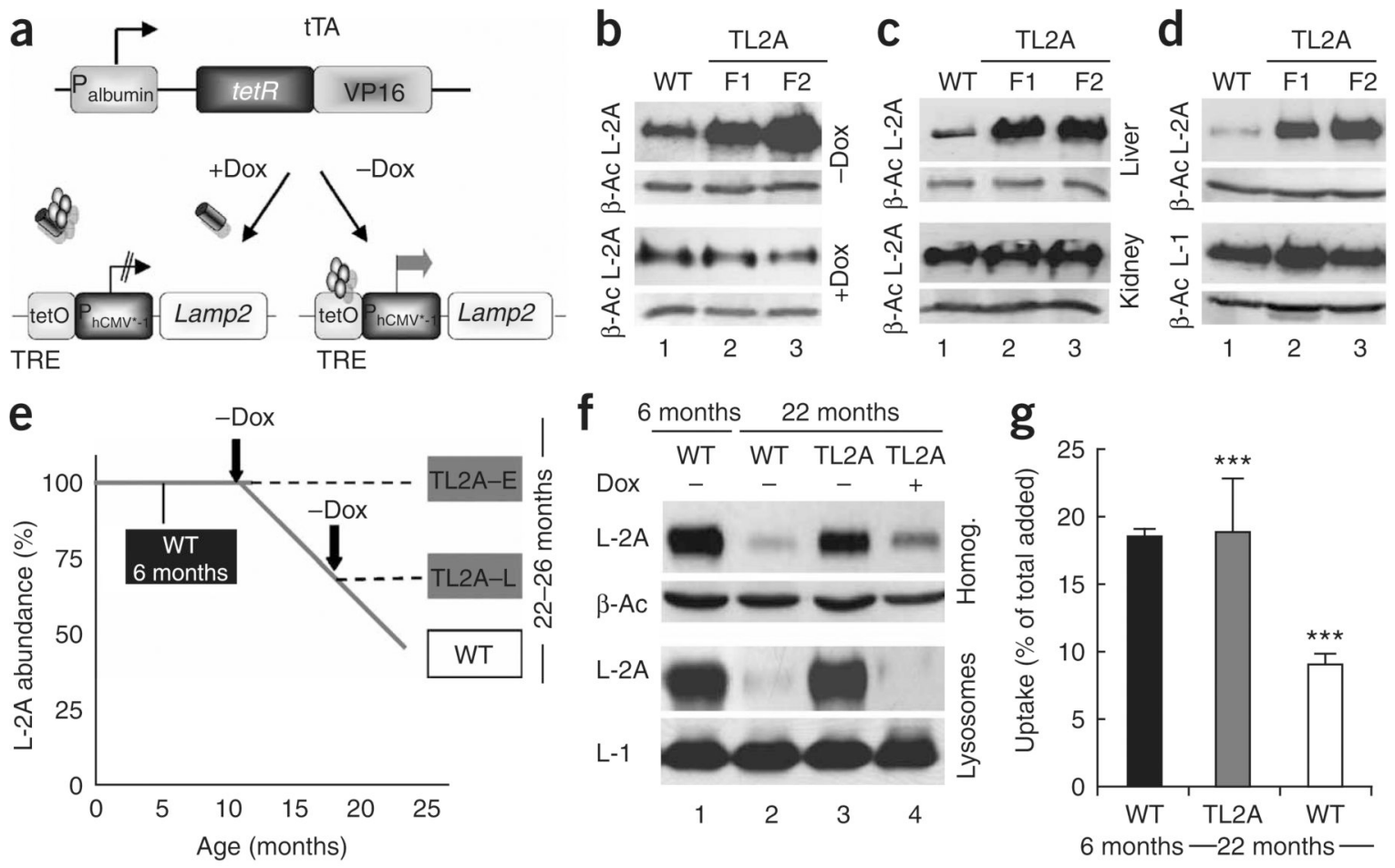

h
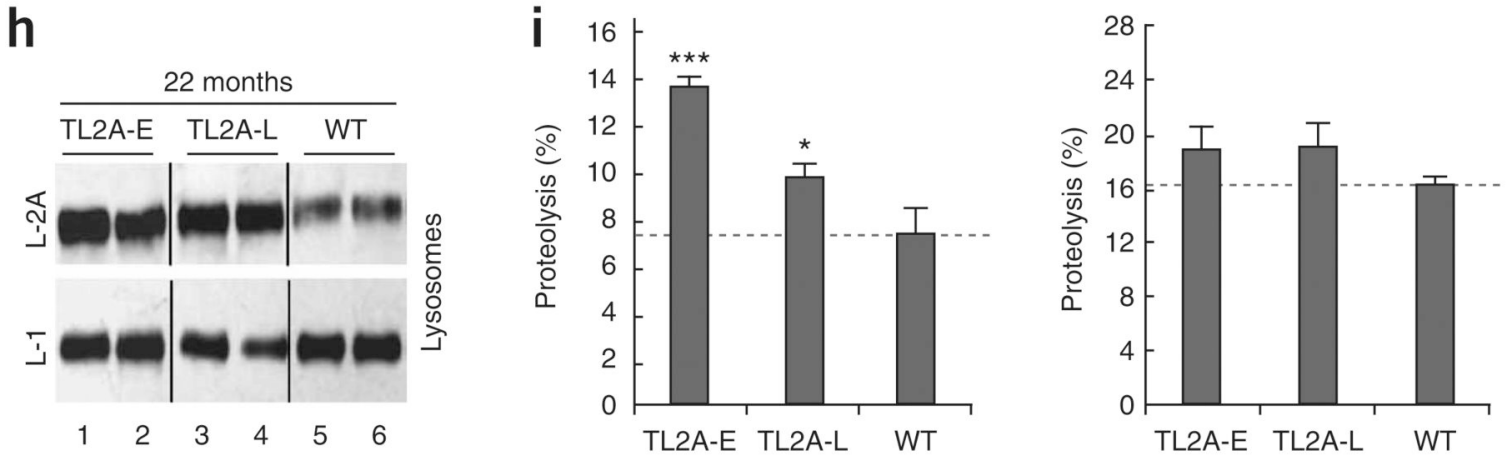

Figure 1.

CMA activity is preserved in livers of aged Alb-Tet-off-L2A mice. (a) Schematic showing that administration of doxycycline prevents transcription of the gene encoding the extra copy of LAMP-2A in the Alb-Tet-off-LAMP-2A mouse. VP16, transactivation domain of the herpes simplex virus protein; tet $\mathrm{O}$, tet operon operator; $\mathrm{PhCMV}^{*}-1$, tetracycline responsive promoter of the human cytomegalovirus. (b) Immunoblot for LAMP-2A (L-2A) or $\beta$-actin $(\beta-A c)$ in liver homogenates from 4-month-old wild-type (WT) or Alb-Tet-off-L2A (TL2A) mice from two different founder lines (F) maintained in the presence of doxycycline food (+Dox) or switched to doxycycline-free food (-Dox) for 4 weeks. (c,d) Immunoblot for LAMP-2A in liver and kidney homogenates (c) or for L-2A and LAMP-1 (L-1) in liver homogenates (d) from similar mouse groups as in $\mathbf{b}$ switched to doxycycline-free food for 4 weeks. (e) Schematic of the groups of mice used in this study. Graph shows the trend of changes in LAMP-2A abundance in liver with age (from our previous studies). TL2A-E, mice with early activation of LAMP-2A; TL2A-L, mice with late activation of LAMP-2A. (f) Immunoblot for L-2A in homogenates (Homog) and lysosomes from 6- or 22-month-old WT mice and 22-month-old TL2A mice maintained or not maintained on a doxycycline diet. (g) Uptake of GAPDH by lysosomes from the same mouse groups as in $\mathbf{f}$. Values are the means \pm s.e.m. of five or six 
experiments. (h) Immunoblot for L-2A and L-1 in lysosomes isolated from 22-month-old WT mice and 22-month-old TL2A-E or TL2A-L mice. (i) Degradation of $\left[{ }^{14} \mathrm{C}\right]$ GAPDH by intact (left) or broken (right) lysosomes isolated from the same mouse groups as in $\mathbf{h}$. Values are the means \pm s.e.m. of three different experiments with duplicate samples. Significant differences between experimental groups and young $\left(^{*}\right)$ or aged (\#) WT mice were: *, $P<0.05$; or ***, $P<0.001$. $\beta$-Ac and L-1 are shown as loading controls for homogenates and lysosomes, respectively. 

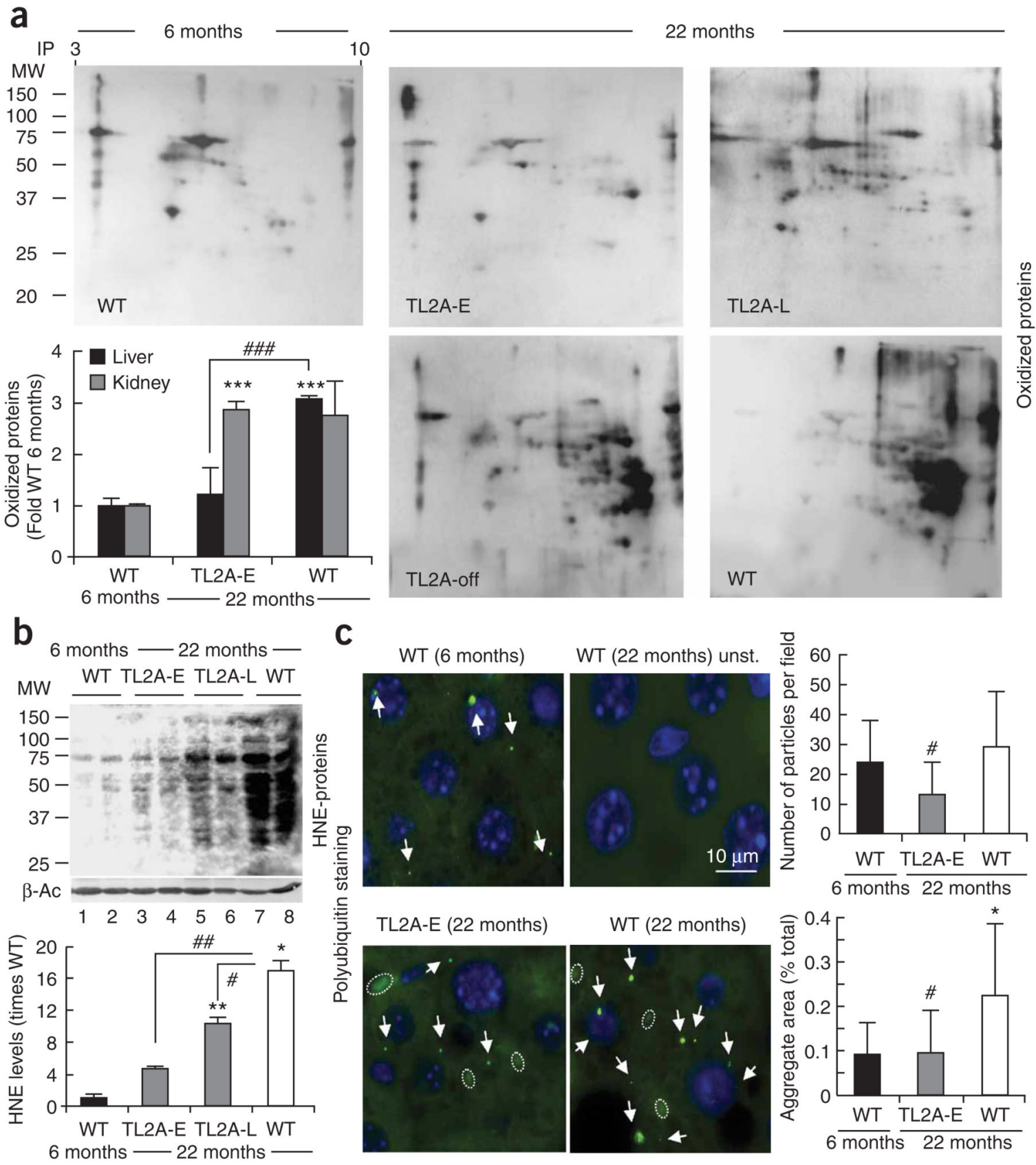

Figure 2.

Livers of aged Alb-Tet-off-L2A mice accumulate fewer damaged proteins. (a) Twodimensional electrophoresis and immunoblotting for carbonyl groups of cytosolic fractions from livers of 6- or 22-month-old WT mice, 22-month-old TL2A-E or TL2A-L mice or transgenic mice maintained on a doxycycline diet throughout their life span (TL2A-off). Loading controls are shown in Supplementary Figure 5a. Graph shows the mean \pm s.e.m. value of the densitometric quantification of two to four immunoblots similar to the ones shown here for liver and in Supplementary Figure 5b for kidney. IP, isoelectric point; MW, molecular weight. (b) Representative immunoblot for 4-hydroxynonenal (4-HNE) in cytosolic fractions from the same groups of mice as in a (top). Actin is shown as a loading control. Densitometric 
quantification of two different experiments, expressed as means \pm s.e.m. (bottom). (c) Aggregate proteins were visualized by fluorescence microscopy as round bright fluorescent puncta in liver sections from the same mouse groups as in $\mathbf{a}$ and $\mathbf{b}$ immunostained for polyubiquitin (profiles of red blood cells have been highlighted with a discontinuous line). An unstained (unst.) section is shown to account for the presence of autofluorescence. At right, the number of aggregates per microscopic field (top) and the percentage of cellular area occupied by the fluorescent puncta (bottom) are shown. Values are means \pm s.e.m. of the morphometric analysis of 10-15 randomly taken pictures of liver sections from four different mice in each group. Significant differences between experimental groups and young $(*)$ or aged (\#) WT mice were: * or \#, $P<0.05$; ** or \#\#, $P<0.01$; or *** or \#\#, $P<0.005$. 
a

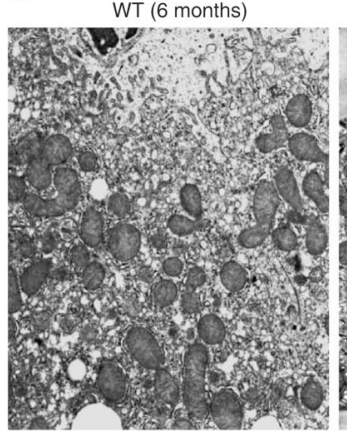

TL2A-L (22 months)

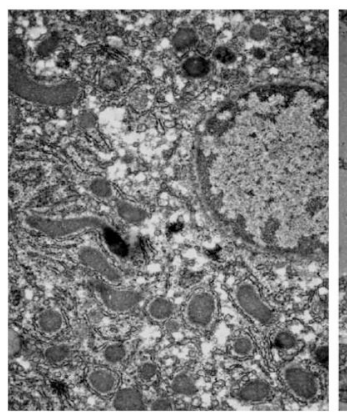

TLA-E (22 months)

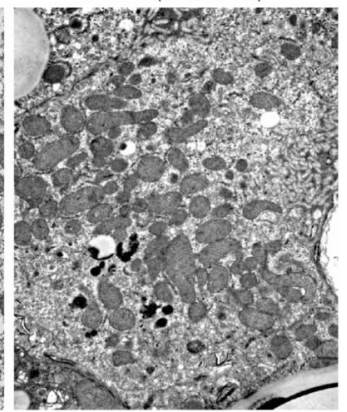

WT (22 months)

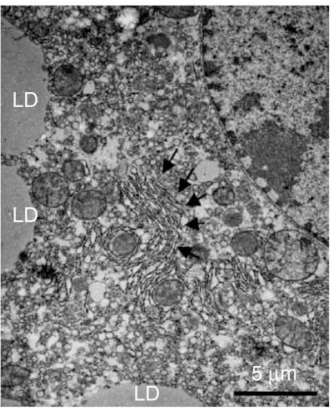

b
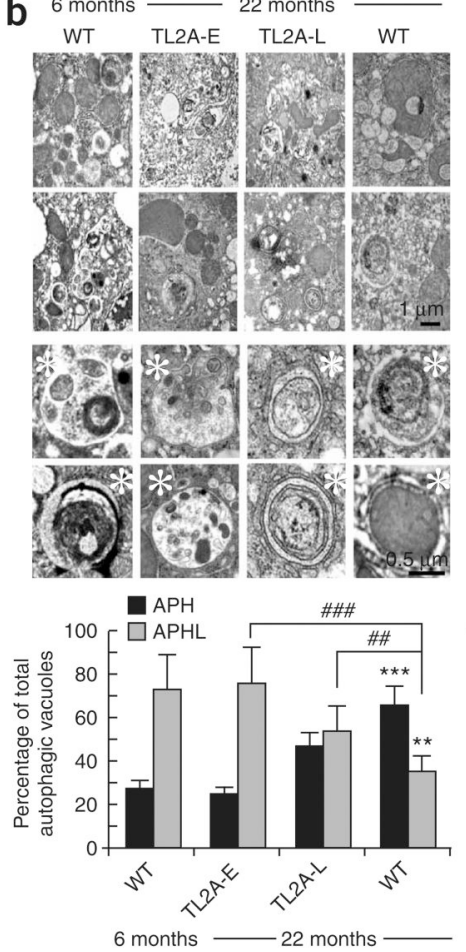

C

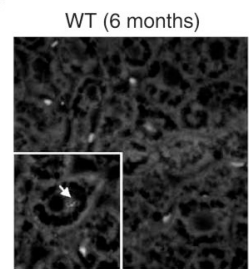

TL2A-L (22 months)
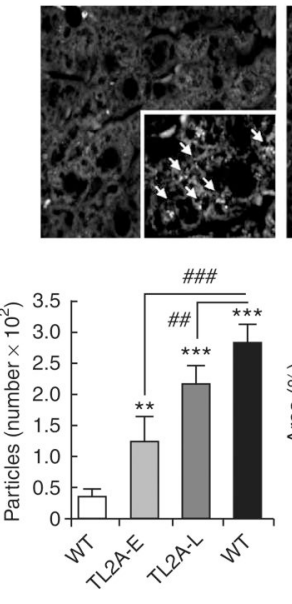

6 months - 22 months -
TL2A-E (22 months)

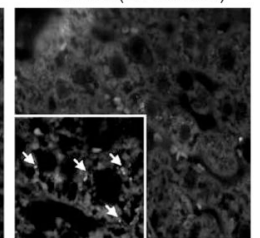

WT (22 months)
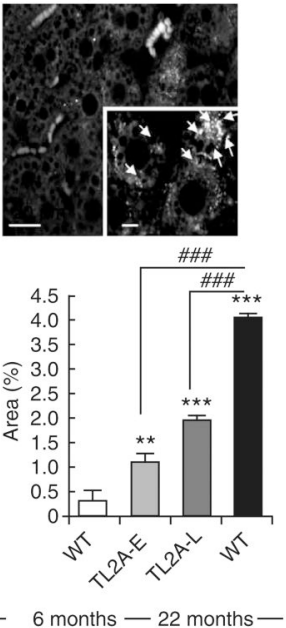

Figure 3.

Improved cellular homeostasis in livers of aged Alb-Tet-off-LAMP-2A mice. (a) Electron micrographs showing ultrastructure of livers from 6- or 220-month-old WT mice or 22-monthold TL2A-E or TL2A-L mice. LD, lipid droplets. Black arrows point to areas of accumulation of endoplasmic reticulum stacks. (b) Higher magnification electron micrographs of the same mouse groups as in a showing areas enriched in autophagosomes and autophagolysosomes (top panels) and representative examples of the two (bottom panels). The percentage of autophagosomes (APH, black asterisk) and autophagolysosomes (APHL, white asterisk) in livers from the different mouse groups (bottom) was calculated after morphometric analysis of seven to ten micrographs for each mouse. Values are expressed as percentage of the total number of autophagic vacuoles and are means \pm s.e.m. of three to five mice in each group. (c) Lipofuscin abundance in livers from the same groups of mice as in $\mathbf{a}$ and $\mathbf{b}$ was detected as autofluorescent puncta when unstained liver sections were observed under a fluorescence microscope. Representative sections with higher magnification areas (insets) are shown at the top. Scale bars, $20 \mu \mathrm{m}$ and $5 \mu \mathrm{m}$ for main images and insets, respectively. The number of particles per cell (left) and the percentage of cellular area occupied by the fluorescent puncta (right) calculated in ten different fields from three different sections in each liver are shown at the bottom. Significant differences between the experimental groups and the young $(*)$ or aged (\#) WT mice were: ** or \#\#, $P<0.05$; or $* * *$ or \#\#\#, $P<0.01$. 


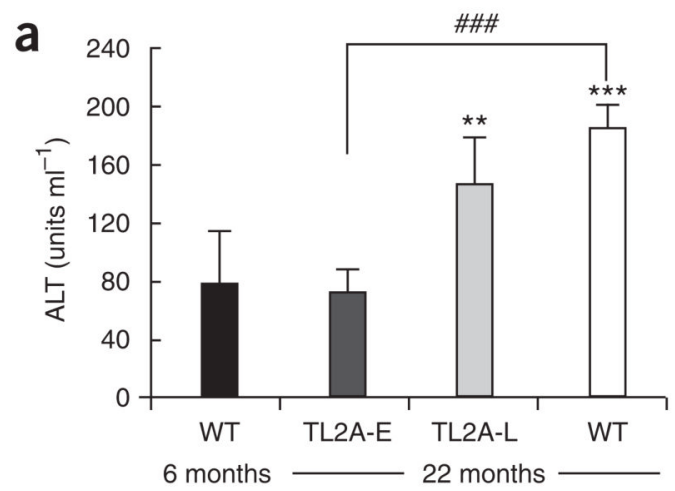

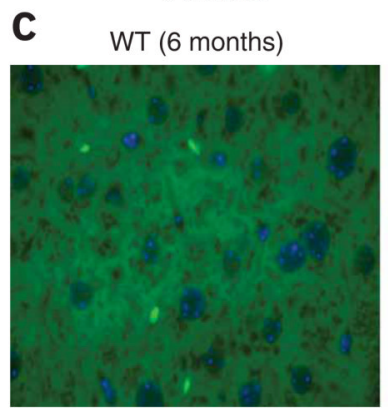

TL2A-L (22 months)

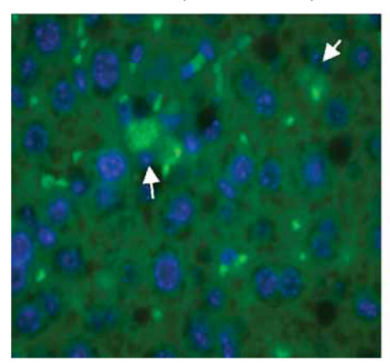

WT (22 months)

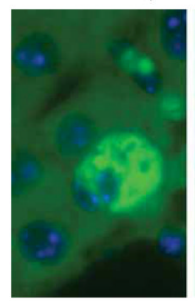

Cytosolic

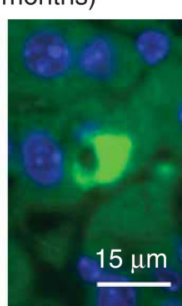

Condensed
TL2A-E (22 months)

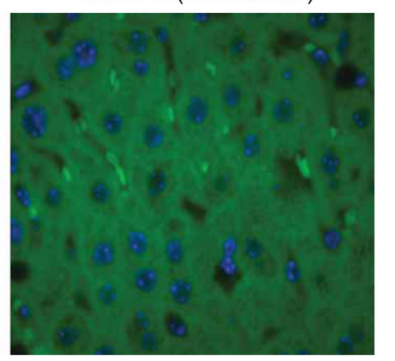

WT (22 months)
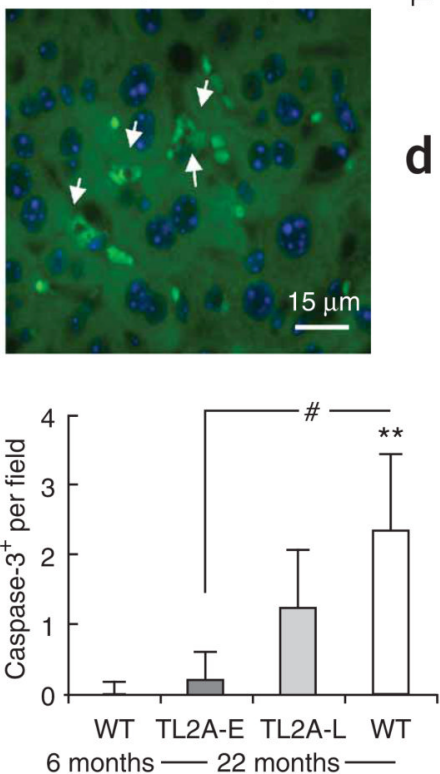

d

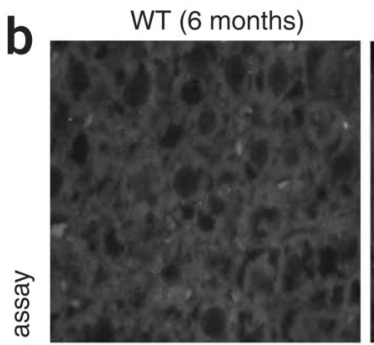

TL2A-E (22 months)

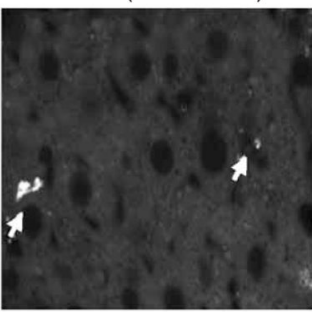

岁

TL2A-L (22 months)

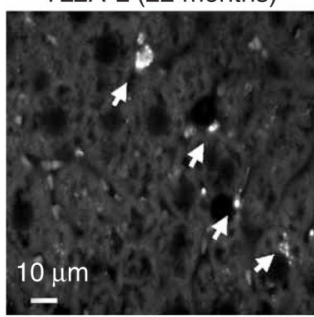

WT (22 months)
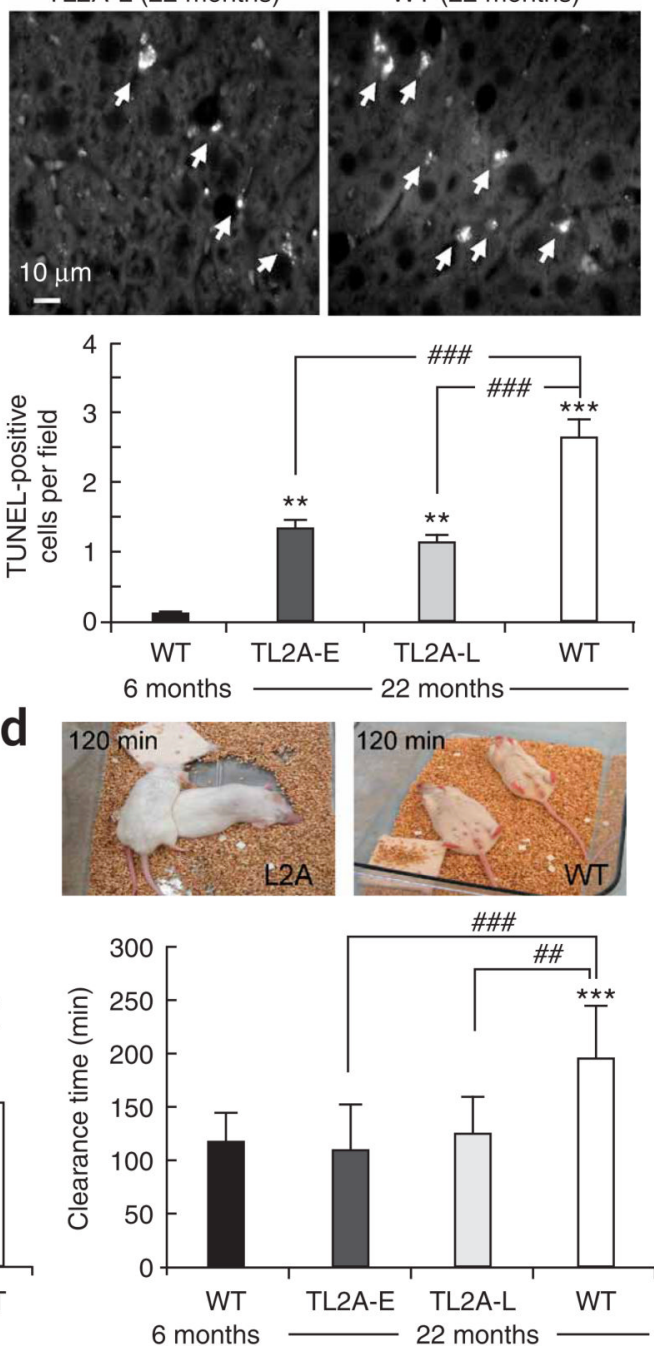

Figure 4.

Livers of aged Alb-Tet-off-L2A mice show lower levels of cellular damage and improved organ function. (a) Serum abundance of alanine aminotransferase (ALT) in 6- or 22-monthold WT mice or 22-month-old TL2A-E or TL2A-L mice. Values are means \pm s.e.m. of six to eight mice in each group. (b,c) TUNEL staining (b) and activated caspase-3

immunofluorescence (c) of liver sections from the same mouse groups as in a. Quantification of the number of TUNEL-positive (b) or activated caspase-3-positive (c) cells per field (means \pm s.e.m. of at least ten different liver sections) is shown in the graphs. Arrows indicate positive cells to differentiate them from the fluorescence of red blood cells in the section. Bottom images in c show examples of the two types of positive cells: cells with cytosolic staining (left) and 
condensed cells probably corresponding to apoptotic bodies (right). (d) Time of recovery from the paralysis induced after injection of the same mouse groups as in a-c with zoxazolamine. Values are means \pm s.e.m. of the recovery times in six to eight mice in each group. Top images show recovery of muscle tone in transgenic aged mice $120 \mathrm{~min}$ after injection. The mice had turned from their backs and started crawling around, whereas the aged WT mice remained immobile, lying on their backs. Significant differences between the experimental groups and the young (*) or aged (\#) WT mice were: \#, $P<0.05$; ** or \#\#, $P<0.01$; or *** or \#\#\#, $P<$ 0.001 . 